\title{
State of the art of identification of eggs and larvae of freshwater fish in Brazil
}

Estado da arte da identificação de ovos e larvas de peixes de água doce no Brasil

\author{
David Augusto Reynalte-Tataje ${ }^{1 *}$ (D), Carolina Antonieta Lopes² (D), \\ Marthoni Vinicius Massaro ${ }^{3}$ (D), Paula Betina Hartmann³ (D), Rosalva Sulzbacher ${ }^{3}$ (D), \\ Joyce Andreia Santos ${ }^{4} \mathbb{D}$ and Andréa Bialetzki ${ }^{5}$
}

${ }^{1}$ Programa de Pós-graduação em Ambiente e Tecnologias Sustentáveis, Universidade Federal da Fronteira Sul - UFFS, Avenida Jacob Reinaldo Haupenthal, 1580, CEP 97900-000, Cerro Largo, RS, Brasil

${ }^{2}$ Programa de Pós-graduação em Aquicultura, Universidade Federal de Santa Catarina - UFSC, Rodovia Admar Gonzaga, 1346, CEP 88034-001, Itacorubi, Florianópolis, SC, Brasil

${ }^{3}$ Universidade Federal da Fronteira Sul - UFFS, Avenida Jacob Reinaldo Haupenthal, 1580, CEP 97900-000, Cerro Largo, RS, Brasil

${ }^{4}$ Programa de Pós-graduação em Ecologia, Instituto de Ciências Biológicas - ICB, Universidade Federal de Juiz de Fora - UFJF, Campos Universitário, CEP 36036-900, Bairro São Pedro, Juiz de Fora, MG, Brasil

${ }^{5}$ Programa de Pós-graduação em Ecologia de Ambientes Aquáticos Continentais, Núcleo de Pesquisas em Limnologia, Ictiologia e Aquicultura - Nupélia, Universidade Estadual de Maringá UEM, Avenida Colombo, 5790, bloco G-80, CEP 87020-900, Maringá, PR, Brasil

*e-mail: david.tataje@uffs.edu.br

Cite as: Reynalte-Tataje, D. A. et al. State of the art of identification of eggs and larvae of freshwater fish in Brazil. Acta Limnologica Brasiliensia, 2020, vol. 32, e6.

Abstract: Aim: This study aimed to assist in guiding research with eggs and larvae of continental fish in Brazil, mainly in the knowledge of the early development, as well as to present the state of the art and to point out the gaps and future directions for the development of researches in the area. Methods: The literature review was carried out in the electronic platforms and studies were examined to investigate whether any addressed morphological description of fish eggs and/or larvae. Results: We found 121 studies, including scientific articles, theses, dissertations, chapters of books and books. This bibliographic production characterized 140 species, representing only $4.1 \%$ of the total species present in the Brazilian basins. Most of the species studied belong to the orders Characiformes and Siluriformes, which are of commercial interest and native to the Paraná, Amazon, Paraguay and Uruguay basins. For some very species-rich families in the Brazilian rivers, such as Characidae and Loricariidade, only a fraction of their species was characterized for the early development (2.6\% and $0.7 \%$ of the total, respectively) and $56 \%$ of the families did not present any study addressing the early ontogeny. Regarding the content of the descriptions, for 73 species, there is characterization of the embryonic period and for 62, there is description of the whole larval period. For only 41 species there were complete descriptions (embryonic and larval) of the early development. All of these studies were conducted mainly with material derived from induced spawning and material obtained in the field (regressive series). Conclusions: The characterization of eggs and larvae, still presents few studies, limited mainly to species from the rivers of the Plata and Amazon basins, to studies of fish of commercial importance and the incompleteness of most of these studies is still outstanding.

Keywords: ichthyoplankton; ontogeny; neotropical region; stages of development. 
Resumo: Objetivo: O objetivo do estudo é auxiliar no direcionamento das pesquisas no Brasil com ovos e larvas de peixes continentais, principalmente quanto ao conhecimento do desenvolvimento inicial, bem como apresentar o estado da arte e apontar as lacunas e direcionamentos futuros para o desenvolvimento das pesquisas na área. Métodos: O levantamento da produção bibliográfica foi realizado através das plataformas eletrônicas e examinadas com o intuito de averiguar se abordava alguma descrição morfológica de ovos e/ou larvas de peixes. Resultados: Foram encontrados 121 estudos, entre artigos científicos, teses, dissertaçôes, capítulos de livros e livros. Nessa produção bibliográfica foram caracterizadas 140 espécies o que representa apenas $4,1 \%$ do total de espécies presentes nas bacias brasileiras. A maior parte das espécies estudadas pertencem às ordens Characiformes e Siluriformes, que apresentam interesse comercial e que são nativas das bacias Paraná, Amazônica, Paraguai e Uruguai. Para algumas famílias muito ricas em espécies nos rios brasileiros como p.e., Characidae e Loricariidade, apenas uma fração de suas espécies foram caracterizadas quanto ao desenvolvimento inicial ( $2,6 \%$ e $0,7 \%$ do total, respectivamente) e $56 \%$ das famílias não apresentam nenhum estudo que aborda a ontogenia inicial. Com relação ao conteúdo das descrições, para 73 espécies há caracterização do período embrionário e 62 apresentaram todo o seu período larval descrito. Apenas para 41 espécies foram encontradas descriçóes completas (embrionário e larval) do desenvolvimento inicial. Todos estes estudos foram realizados principalmente, com material proveniente de desova induzida e material obtido em campo (série regressiva). Conclusóes: Podemos concluir que a caracterização de ovos e larvas apresenta ainda poucos estudos, limitados principalmente a espécies que habitam os rios da Bacia do Plata e Amazônica, aos estudos de peixes de importância comercial e onde se destaca ainda a incompletude da maior parte destes trabalhos.

Palavras-chaves: ictioplâncton; ontogenia; região neotropical; estágios de desenvolvimento.

\section{Introduction}

Accurate identification of the early stages of fish development is an important step in the study of fish ecology or fishery biology, however, due to the drastic morphological transformations (metamorphosis) that most species suffer from the earliest larval stages to juvenile formation, many individuals are erroneously identified.

In continental environments, the initial development of fish is a process that can last for days or weeks and larvae generally have between 1.0 and $8.0 \mathrm{~mm}$ (total length) at hatching, and can reach until the end of its development (juvenile period) between 10 and $40 \mathrm{~mm}$ (Nakatani et al., 2001; Reynalte-Tataje \& Zaniboni-Filho, 2008). During this period, larvae often have few similarities with adults, for two reasons: (i) either because they are partially or incompletely developed, they do not have the same morphological structures found in adults, such as Pimelodidae larvae, which upon hatching usually do not present barbells, characteristic of the adults of this family, or (ii) because they present specializations totally different from their parents, resulting in the presence of morphological characteristics that resemble very little or nothing at all the adults (Nakatani et al., 2001), as is the case of the darter characin, Apareiodon affinis (Steindachner, 1879), which has terminal mouth at the beginning of the larval stage when it feeds on algae, and inferior mouth, in the juvenile period, when it begins to feed on periphyton (Santin et al., 2009).

In Brazil, despite having one of the highest diversity of freshwater fish species in the world (Froese \& Pauly, 2018), studies on egg systematics are almost non-existent and on larvae, are incipient and only for few species their initial stages of development are known (Bialetzki et al., 2016). In most cases, morphological descriptions are of a specific nature, and are scattered in isolated and/or of restricted access publications and are often incomplete (Nakatani et al., 2001). Thus, the objective of this study was to perform a scientometric analysis of the studies involving the characterization of the early development of freshwater fish in Brazil. Specifically, it is intended: (i) to survey the means of dissemination of scientific production, as well as their periodicity; (ii) to evaluate the number of studies per hydrographic region; (iii) to know the species whose early morphological development was described in the literature and (iv) to check the methods most used to obtain material for such studies. Our expectation is to present the state of the art on the knowledge of the early ontogeny of the Brazilian continental species, as well as to point out the gaps and future directions for the advance of researches in the area.

\section{Material and Methods}

The study was performed between November 2018 and February 2019 in ichthyoplankton biology books and electronic platforms: Periódicos Capes, Science Direct and Google Scholar, and all the bibliographic production found was examined to check for information on the early development of eggs and/or larvae of fish native to Brazil.

The key-words used in the searches in English, Portuguese and Spanish were: "ontogenetic", "embryonic development", "early life fish" and "larval 
development", "Neotropical freshwater", besides the Brazilian hydrographic regions established by the National Council of Hydrographic Resources CNRH (Brasil, 2003): Amazon, Tocantins/Araguaia, Western Northeast Atlantic, Eastern Northeast Atlantic, Parnaíba, East Atlantic, São Francisco, Southeast Atlantic, South Atlantic, Paraná, Uruguay and Paraguay hydrographic regions.

Each reference was analyzed and inserted in a spreadsheet which included the following information: type of production (scientific article, dissertation or thesis and chapter of book or book), year of publication, species name, family and order (Reis et al., 2003; Fricke et al., 2019), hydrographic region of origin, commercial importance (Godinho, 2007; Baldisserotto \& Gomes 2010; Valladão et al., 2018) and the presence or absence of images (photos and/or illustrations), as well as the presence or absence of images (photos and/or illustrations) of eggs or larvae at any stage of development (yolk-sac, pre-flexion, flexion or post-flexion; sensu Nakatani et al., 2001).
Information on the methodology used to obtain the collections were also extracted and classified into RS - regressive series (material obtained in the field; but fixed), LI - live ichthyoplankton (material obtained in the field and kept fresh) and IS - induced spawning.

\section{Results}

In the electronic platforms, from 1977 to 2018 , we found 121 references among scientific articles, dissertations, theses, chapters of books and books related to the morphological characterization of freshwater fish eggs and/or larvae of Brazil. Most of the studies were published as a scientific article (Figure 1) and over the years, there was a growing increase in the number of publications, significant from the year 2009 (Figure 2) although the first peak of production occurred in 2001.

Most of the studies were developed with species inhabiting the hydrographic regions of the South and Southeast of Brazil, such as those of the Paraná, Paraguay and Uruguay basins, with 70, 46 and 45 species,

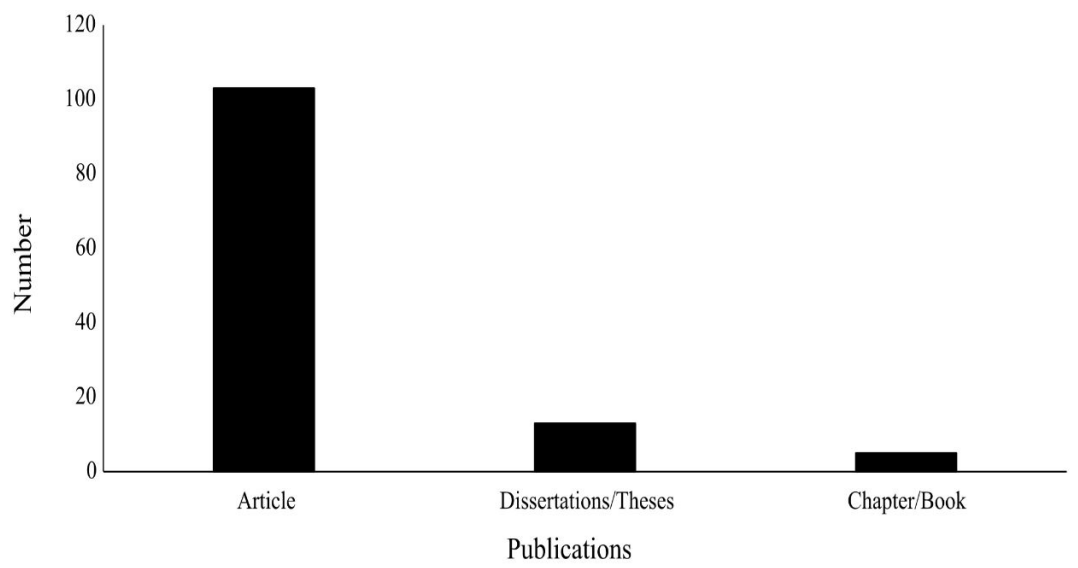

Figure 1. Types of bibliographic production containing morphological descriptions of eggs and/or larvae of freshwater fish in Brazil produced between 1977 and 2018.

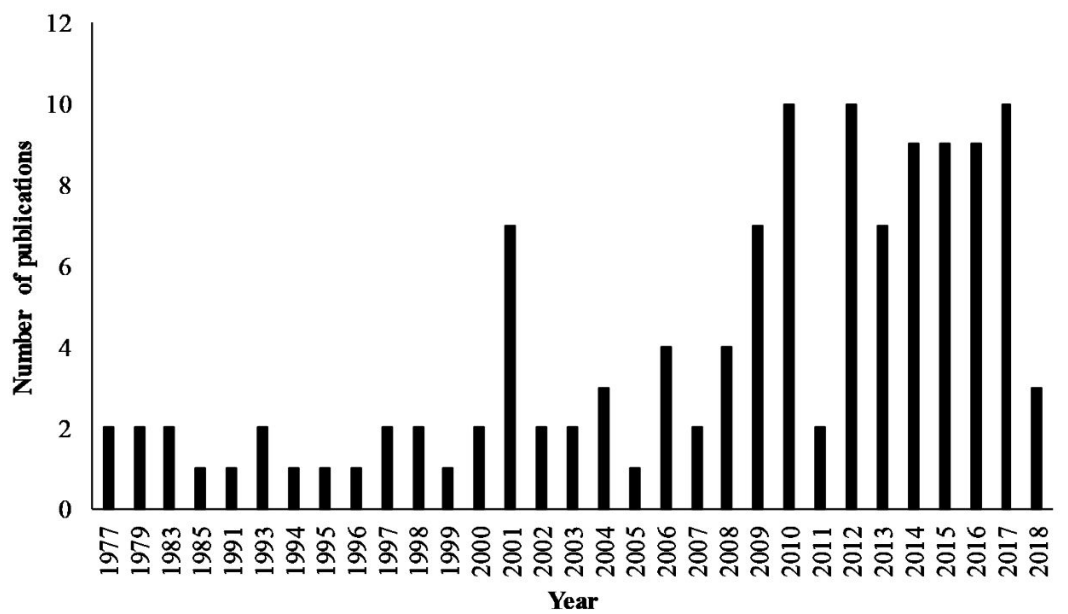

Figure 2. Number of publications produced containing morphological descriptions of eggs and larvae of freshwater fish in Brazil between 1977 and 2018. 
respectively. The Amazon basin, with 59 species, was the second region in number of studies with morphological characterization (Figure 3).

Studies on the morphological characterization of the early development of fish were carried out with 140 species, most belonging to the order Characiformes (74 species) and Siluriformes (48 species). For some species, more than one study was found, such as Prochilodus lineatus (Valenciennes, 1837; eight studies) and Pimelodus maculatus (Lacepède, 1803; nine studies). Among the species studied, 51 (36.0\%) are of commercial importance (Table 1).

By relating the number of species that have their early development described (140) with the number of species described for the same family, it is observed that for the great majority of freshwater fish families in Brazil there is a huge gap in the studies. For some families with a high number of species such as Characidae and Loricariidae, only a fraction of their species was described for the early development $(2.6 \%$ and $0.7 \%$ of the total, respectively). It is still possible to verify that of the 67 fish families present in Brazilian continental waters, more than $56.0 \%$ do not present any study that approaches the early ontogeny (Figure 4).

Regarding the content of the descriptions, only for 73 species there is characterization of the embryonic period. On the other hand, for 66 species, there is characterization of at least one of their larval developmental stages, and for 62, there is description of the whole larval period. Only for 41 species, most with some commercial interest, there are complete descriptions (embryonic and larval) of the early development (Table 1 ). All these studies were carried out mainly with material from induced spawning and regressive series (Table 1).

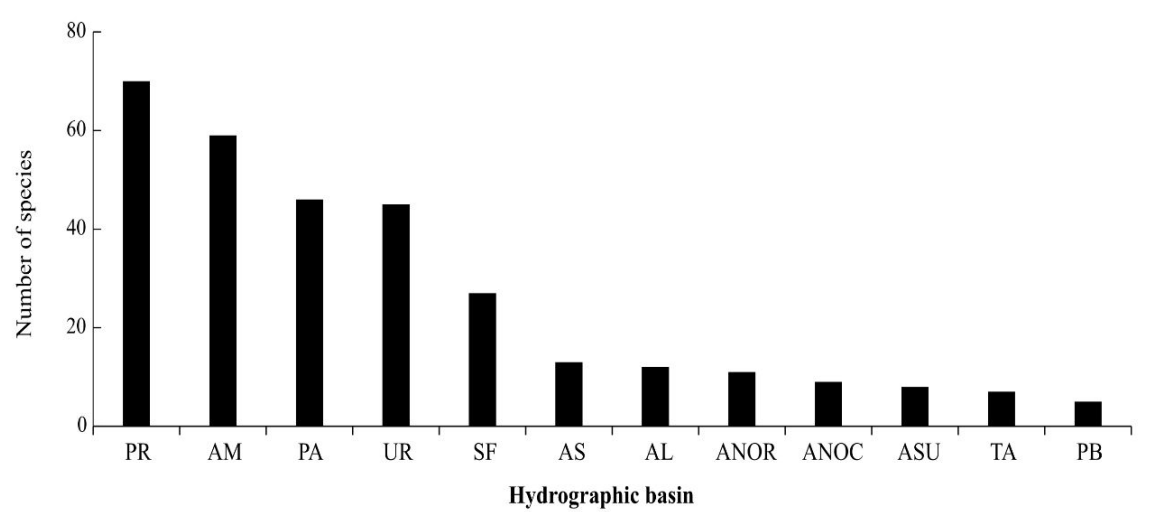

Figure 3. Number of freshwater fish species described as to their early development (eggs and/or larvae) in the different Brazilian river basins between 1977 and 2018. PR: Paraná; AM: Amazon; PA: Paraguay; UR: Uruguay; SF: São Francisco; AS: Southeast Atlantic; AL: East Atlantic; ANOR: Eastern Northeast Atlantic; ANOC: Western Northeast Atlantic; ASU: South Atlantic; TA: Tocantins-Araguaia and PB: Parnaíba.

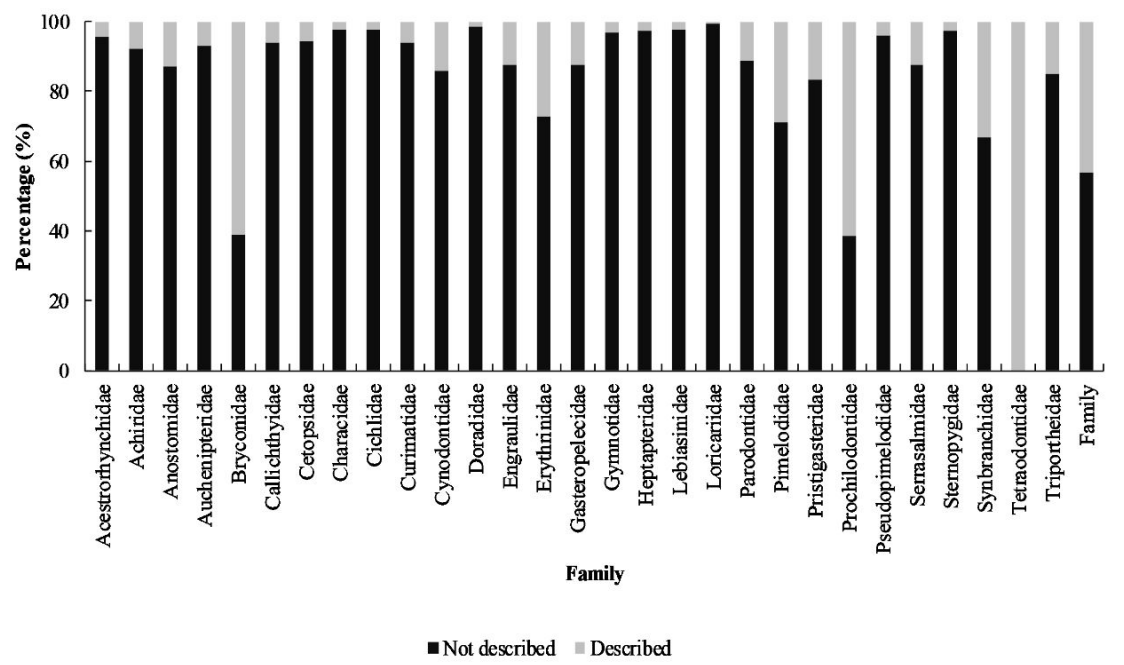

Figure 4. Fish families present in Brazilian continental waters and percentage of the number of species with early development described in the literature. 
Table 1. Brazilian continental fish species with characterization of their early development.

\begin{tabular}{|c|c|c|c|c|c|}
\hline \multirow{2}{*}{ Species } & \multirow{2}{*}{ HR } & \multirow{2}{*}{ Egg } & \multicolumn{2}{|c|}{ Larvae } & \multirow{2}{*}{ MU } \\
\hline & & & $\mathbf{L}$ & C & \\
\hline \multicolumn{6}{|l|}{ CHARACIFORMES } \\
\hline \multicolumn{6}{|l|}{ Acestrorhynchidae } \\
\hline Heterocharax macrolepis Eigenmann, 1912 & a & & $x$ & & IS \\
\hline \multicolumn{6}{|l|}{ Anostomidae } \\
\hline Leporinus agassizii Steindachner, 1876 & a & $\mathrm{x}$ & & & IS \\
\hline Leporinus amblyrhynchus Garavello \& Britski, 1987 & $\mathrm{~h}$ & & $x$ & & RS \\
\hline Leporinus copelandii Steindachner, 1875 & e & $\mathrm{X}$ & & & IS \\
\hline Leporinus friderici (Bloch, 1794) & a & $\mathrm{X}$ & & $\mathrm{X}$ & IS/RS \\
\hline Leporinus octofasciatus Steindachner, 1915 & $\mathrm{~h}$ & $x$ & & & RS \\
\hline Leporinus piau Fowler, 1941 & $\mathrm{c}$ & $\mathrm{x}$ & & & IS \\
\hline Leporinus steindachneri Eigenmann, 1907 & d & $x$ & $x$ & & IS \\
\hline Megaleporinus piavussu (Britski, Birindelli \& Garavello, 2012) & $\mathrm{h}$ & & $\mathrm{X}$ & & RS \\
\hline Megaleporinus macrocephalus (Garavello \& Britski, 1988) & $g$ & $\mathrm{X}$ & & $\mathrm{X}$ & IS \\
\hline Megaleporinus obtusidens (Valenciennes, 1837$)^{\star}$ & $f, g, h, i, j, l$ & $\mathrm{x}$ & & $\mathrm{X}$ & IS/RS \\
\hline Megaleporinus elongatus (Valenciennes, 1850$)^{\star}$ & d & $\mathrm{X}$ & $x$ & & IS \\
\hline Schizodon borellii (Boulenger, 1900) & $g$ & $\mathrm{X}$ & $\mathrm{X}$ & & IS \\
\hline Schizodon fasciatus Spix \& Agassiz, 1829 & a & $\mathrm{X}$ & & $x$ & IS \\
\hline Schizodon nasutus Kner, 1858 & $\mathrm{~g}, \mathrm{~h}, \mathrm{l}$ & $x$ & & $x$ & IS \\
\hline \multicolumn{6}{|l|}{ Bryconidae } \\
\hline Brycon amazonicus (Agassiz, 1829)* & a & $\mathrm{X}$ & $x$ & & IS \\
\hline Brycon gouldingi Lima, 2004 & k & $x$ & $\mathrm{X}$ & & IS \\
\hline Brycon hilarii (Valenciennes, 1850) & $a, g, h$ & & & $x$ & RS \\
\hline Brycon insignis Steindachner, 1877 & e & $x$ & & $x$ & RS \\
\hline Brycon nattereri Günther, 1864 & $h, j$ & $x$ & & $x$ & IS \\
\hline Brycon orbignyanus (Valenciennes, 1850) & $\mathrm{h}, \mathrm{l}$ & $\mathrm{X}$ & & $x$ & IS \\
\hline Brycon orthotaenia Günther, 1864 & j & $\mathrm{x}$ & & $\mathrm{X}$ & IS \\
\hline Salminus brasiliensis (Cuvier, 1816)* & $a, g, h, I$ & $\mathrm{X}$ & & $x$ & \\
\hline Salminus franciscanus Lima \& Britski, $2007^{*}$ & j & $x$ & & $x$ & IS \\
\hline Salminus hilarii Valenciennes, 1850 & $a, h, j, k$ & $\mathrm{X}$ & & $x$ & IS \\
\hline \multicolumn{6}{|l|}{ Characidae } \\
\hline Aphyocharax cf. anisitsi & h,l & & $x$ & & RS \\
\hline Astyanax lacustris (Lütken, 1875) & $e, f, h, I$ & $\mathrm{X}$ & & $x$ & RS \\
\hline Astyanax gr. scabripinnis & d & $x$ & & & RS \\
\hline Bryconamericus iheringii (Boulenger, 1887) & $f, g, I$ & & & $\mathrm{X}$ & $\mathrm{RS} / \mathrm{LI}$ \\
\hline Gymnocorymbus ternetzi (Boulenger, 1895) & g & $x$ & & $\mathrm{X}$ & IS \\
\hline Hemigrammus marginatus Ellis, 1911 & $a, d, g, h, j$ & & $x$ & & RS \\
\hline Hyphessobrycon anisitsi (Eigenmann, 1907) & h,l & $x$ & & $\mathrm{X}$ & IS \\
\hline Hyphessobrycon eques (Steindachner, 1882) & $a, h$ & & & $x$ & IS/RS \\
\hline Knodus moenkhausii (Eigenmann \& Kennedy, 1903) & a & & $x$ & & RS \\
\hline Moenkhausia cf. gracilima & A & & $\mathrm{X}$ & & RS \\
\hline Moenkhausia intermedia Eigenmann, 1908 & $a, g, h, l$ & & $x$ & & RS \\
\hline Moenkhausia pittieri Eigenmann, 1920 & & & & $\mathrm{X}$ & IS \\
\hline Moenkhausia sanctaefilomenae (Steindachner, 1907) & $e, g, h, j, l$ & $\mathrm{X}$ & & $x$ & \\
\hline Paracheirodon axelrodi (Schultz, 1956) & a & $\mathrm{X}$ & $x$ & & IS \\
\hline Paracheirodon innesi (Myers, 1936) & a & & & $\mathrm{X}$ & IS \\
\hline Piabarchus stramineus (Eigenmann, 1908) & $g, h, j, I$ & & $x$ & & RS \\
\hline Roeboides descalvadensis Fowler, 1932 & $\mathrm{a}, \mathrm{g}, \mathrm{h}$ & & $\mathrm{X}$ & & RS \\
\hline \multicolumn{6}{|l|}{ Curimatidae } \\
\hline Curimatella lepidura (Eigenmann \& Eigenmann, 1889) & j & $\mathrm{X}$ & & & \\
\hline Potamorhina altamazonica (Cope, 1878) & a & & $\mathrm{X}$ & & IS \\
\hline
\end{tabular}

HR: Hydrographic region of origin, $\mathrm{a}=$ Amazon, $\mathrm{b}=$ Western Northeast Atlantic, $\mathrm{c}=$ Eastern Northeast Atlantic, $\mathrm{d}=$ East Atlantic, $\mathrm{e}=$ Southeast Atlantic, $\mathrm{f}=$ South Atlantic, $\mathrm{g}=$ Paraguay, $\mathrm{h}=$ Paraná, $\mathrm{i}=$ Parnaíba, $\mathrm{j}=$ São Francisco, $\mathrm{k}=$ Tocantins-Araguaia and $\mathrm{i}=$ Uruguay; Larvae, $\mathrm{I}=$ Incomplete, without all larval stages and $\mathrm{C}=$ Complete, with all larval stages; $\mathrm{MU}=$ Methodology used to obtain the collection, $\mathrm{RS}=$ regressive series, $\mathrm{IS}=$ induced spawning and $\mathrm{LI}=$ live ichthyoplankton * commercial species. 
Table 1. Continued...

\begin{tabular}{|c|c|c|c|c|c|}
\hline \multirow{2}{*}{ Species } & \multirow{2}{*}{ HR } & \multirow{2}{*}{ Egg } & \multicolumn{2}{|c|}{ Larvae } & \multirow{2}{*}{ MU } \\
\hline & & & $\mathbf{L}$ & C & \\
\hline Psectrogaster amazonica Eigenmann \& Eigenmann, 1889 & $\mathrm{a}, \mathrm{k}$ & & & $\mathrm{X}$ & IS \\
\hline Steindachnerina brevipinna (Eigenmann \& Eigenmann, 1889) & $\mathrm{g}, \mathrm{h}, \mathrm{l}$ & $\mathrm{X}$ & $\mathrm{X}$ & & IS \\
\hline Steindachnerina elegans (Steindachner, 1875) & $d, j$ & $x$ & & & \\
\hline \multicolumn{6}{|l|}{ Cynodontidae } \\
\hline Rhaphiodon vulpinus Spix \& Agassiz, 1829 & $a, g, h, l$ & & & $\mathrm{X}$ & IS \\
\hline \multicolumn{6}{|l|}{ Erytrinidae } \\
\hline Hoplerythrinus unitaeniatus (Spix \& Agassiz, 1829) & $a, h, j$ & $\mathrm{X}$ & & & IS \\
\hline Hoplias aff. malabaricus* & $b, c$ & $\mathrm{X}$ & $\mathrm{X}$ & & RS \\
\hline Hoplias lacerdae Miranda Ribeiro, 1908 & $e, h, l$ & $\mathrm{X}$ & & $\mathrm{X}$ & IS/RS \\
\hline \multicolumn{6}{|l|}{ Gasteropelecidae } \\
\hline Thoracocharax stellatus (Kner, 1858) & $\mathrm{a}, \mathrm{h}$ & & $\mathrm{X}$ & & RS \\
\hline \multicolumn{6}{|l|}{ Lebiasinidae } \\
\hline Pyrrhulina australis Eigenmann \& Kennedy, 1903 & g,h,l & & $\mathrm{X}$ & & IS \\
\hline \multicolumn{6}{|l|}{ Parodontidae } \\
\hline Apareiodon affinis (Steindachner, 1879) & $\mathrm{g}, \mathrm{h}, \mathrm{l}$ & & $\mathrm{X}$ & & $\mathrm{RS} / \mathrm{LI}$ \\
\hline Apareiodon vittatus Garavello, 1977 & $\mathrm{~h}$ & & & $\mathrm{X}$ & RS \\
\hline \multicolumn{6}{|l|}{ Prochilodontidae } \\
\hline Prochilodus argenteus Spix \& Agassiz, 1829* & j & $\mathrm{X}$ & & $\mathrm{X}$ & IS \\
\hline Prochilodus brevis Steindachner, $1875^{\star}$ & $b, c, j$ & $\mathrm{X}$ & & $\mathrm{X}$ & IS \\
\hline Prochilodus costatus Valenciennes, $1850^{*}$ & $d, j$ & $\mathrm{X}$ & & $x$ & IS \\
\hline Prochilodus harttii Steindachner, $1875^{\star}$ & $d$ & $\mathrm{X}$ & $X$ & & IS \\
\hline Prochilodus lineatus (Valenciennes, 1837)* & $e, g, h, l$ & $\mathrm{x}$ & & $\mathrm{x}$ & IS/RS \\
\hline Prochilodus vimboides Kner, 1859* & $\mathrm{d}, \mathrm{e}$ & $\mathrm{x}$ & & $\mathrm{X}$ & IS \\
\hline Semaprochilodus insignis (Jardine, 1841)* & a & & $\mathrm{X}$ & & RS \\
\hline \multicolumn{6}{|l|}{ Serrasalmidae } \\
\hline Colossoma macropomum (Cuvier, 1816)* & a & $\mathrm{X}$ & & $\mathrm{x}$ & IS \\
\hline Mylossoma duriventre (Cuvier, 1818) & a & & $\mathrm{X}$ & & RS \\
\hline Myloplus tiete (Eigenmann \& Norris, 1900) & $\mathrm{g}, \mathrm{h}$ & & $\mathrm{X}$ & & RS \\
\hline Piaractus brachypomus (Cuvier, 1818)* & a & $\mathrm{X}$ & $\mathrm{X}$ & & IS \\
\hline Piaractus mesopotamicus (Holmberg, 1887)* & $\mathrm{g}, \mathrm{h}, \mathrm{l}$ & $\mathrm{x}$ & & $x$ & IS/RS \\
\hline Serrasalmus brandtii Lütken, 1875 & j & & $\mathrm{X}$ & & RS \\
\hline Serrasalmus maculatus Kner, 1858 & $a, g, h, l$ & & $\mathrm{X}$ & & RS \\
\hline Serrasalmus marginatus Valenciennes, 1837 & g,h & & & $\mathrm{X}$ & RS \\
\hline Serrasalmus spilopleura Kner, 1858 & a & & & $\mathrm{X}$ & RS \\
\hline \multicolumn{6}{|l|}{ Triportheidae } \\
\hline Triportheus angulatus (Spix \& Agassiz, 1829) & a & & $\mathrm{X}$ & & RS \\
\hline Triportheus cf. signatus & $b, c, e$ & $x$ & $x$ & & IS \\
\hline Triportheus nematurus (Kner, 1858) & g,h & & $x$ & & RS \\
\hline \multicolumn{6}{|l|}{ CICHLIFORMES } \\
\hline \multicolumn{6}{|l|}{ Cichlidae } \\
\hline Apistogramma cacatuoides Hoedeman, 1951 & a & & $\mathrm{X}$ & & IS \\
\hline Astronotus ocellatus (Agassiz, 1831) & a & $\mathrm{X}$ & & $\mathrm{X}$ & IS \\
\hline Cichlasoma dimerus (Heckel, 1840) & $\mathrm{g}, \mathrm{h}, \mathrm{l}$ & $\mathrm{x}$ & & $\mathrm{X}$ & IS \\
\hline Crenicichla vittata Heckel, 1840 & g,h,l & $x$ & $\mathrm{X}$ & & $\mathrm{LI}$ \\
\hline Satanoperca pappaterra (Heckel, 1840) & $\mathrm{a}, \mathrm{h}$ & $\mathrm{X}$ & & $\mathrm{X}$ & RS \\
\hline Symphysodon aequifasciatus Pellegrin, 1904 & a & $x$ & & & IS \\
\hline \multicolumn{6}{|l|}{ CLUPEIFORMES } \\
\hline \multicolumn{6}{|l|}{ Engraulidae } \\
\hline Anchoviella vaillanti (Steindachner, 1908) & j & & & $\mathrm{x}$ & RS \\
\hline Lycengraulis grossidens (Spix \& Agassiz, 1829) & $\mathrm{f}, \mathrm{I}$ & & $\mathrm{X}$ & & RS \\
\hline
\end{tabular}

HR: Hydrographic region of origin, $\mathrm{a}=$ Amazon, $\mathrm{b}=$ Western Northeast Atlantic, c $=$ Eastern Northeast Atlantic, $\mathrm{d}=$ East Atlantic, $\mathrm{e}=$ Southeast Atlantic, $\mathrm{f}=$ South Atlantic, $\mathrm{g}=$ Paraguay, $\mathrm{h}=$ Paraná, $\mathrm{i}=$ Parnaíba, $\mathrm{j}=$ São Francisco, $\mathrm{k}=$ Tocantins-Araguaia and $\mathrm{i}=$ Uruguay; Larvae, $\mathrm{I}=$ Incomplete, without all larval stages and $\mathrm{C}=$ Complete, with all larval stages; $\mathrm{MU}=$ Methodology used to obtain the collection, $\mathrm{RS}=$ regressive series, $\mathrm{IS}=$ induced spawning and LI = live ichthyoplankton * commercial species. 
Table 1. Continued...

\begin{tabular}{|c|c|c|c|c|c|}
\hline \multirow{2}{*}{ Species } & \multirow{2}{*}{ HR } & \multirow{2}{*}{ Egg } & \multicolumn{2}{|c|}{ Larvae } & \multirow{2}{*}{ MU } \\
\hline & & & $\mathbf{L}$ & $\mathbf{C}$ & \\
\hline \multicolumn{6}{|l|}{ Pristigasteridae } \\
\hline Pellona flavipinnis (Valenciennes, 1837) & a & & $\mathrm{X}$ & & RS \\
\hline \multicolumn{6}{|l|}{ GYMNOTIFORMES } \\
\hline \multicolumn{6}{|l|}{ Gymnotidae } \\
\hline Gymnotus cf. carapo & $g$ & & & $\mathrm{X}$ & RS \\
\hline \multicolumn{6}{|l|}{ Sternopygidae } \\
\hline Eigenmannia trilineata López \& Castello, 1966 & $\mathrm{~g}, \mathrm{~h}, \mathrm{l}$ & & & $\mathrm{X}$ & RS \\
\hline Eigenmannia virescens (Valenciennes, 1836) & g,h,l & & $\mathrm{X}$ & & RS \\
\hline \multicolumn{6}{|l|}{ PERCIFORMES } \\
\hline \multicolumn{6}{|l|}{ Sciaenidae } \\
\hline Pachyurus bonariensis Steindachner, 1879 & g,h,l & & & $x$ & RS \\
\hline Plagioscion squamosissimus (Heckel, 1840) & $a, g, h, j$ & & $\mathrm{X}$ & & RS \\
\hline Plagioscion ternetzi Boulenger, 1895* & $\mathrm{g}, \mathrm{h}$ & & & $\mathrm{X}$ & RS \\
\hline \multicolumn{6}{|l|}{ PLEURONECTIFORMES } \\
\hline \multicolumn{6}{|l|}{ Achiridae } \\
\hline Catathyridium jenynsii (Günther, 1862) & $\mathrm{h}, \mathrm{l}$ & & $\mathrm{X}$ & & RS \\
\hline \multicolumn{6}{|l|}{ SILURIFORMES } \\
\hline \multicolumn{6}{|l|}{ Auchenipteridae } \\
\hline Ageneiosus inermis (Linnaeus, 1766) & $a, b, c, d, e, f, g, h, i, j, k, l$, & $\mathrm{X}$ & $\mathrm{X}$ & & RS \\
\hline Auchenipterus osteomystax (Miranda Ribeiro, 1918) & $\mathrm{a}, \mathrm{g}, \mathrm{h}, \mathrm{k}, \mathrm{l}$ & & $\mathrm{X}$ & & RS \\
\hline Tatia intermedia (Steindachner, 1877) & a & & $x$ & & RS \\
\hline Tatia jaracatia Pavanelli \& Bifi, 2009 & $\mathrm{~h}$ & & $\mathrm{X}$ & & RS \\
\hline Tatia neivai (Ihering, 1930) & g & & & $\mathrm{X}$ & RS \\
\hline Trachelyopterus galeatus (Linnaeus, 1766) & $a, b, c, d, e, f, g, h, i, j, k, l$, & $\mathrm{X}$ & & $\mathrm{X}$ & IS/RS \\
\hline \multicolumn{6}{|l|}{ Callichthyidae } \\
\hline Corydoras aeneus (Gill, 1858) & $\mathrm{g}, \mathrm{h}, \mathrm{l}$ & & & $\mathrm{X}$ & IS \\
\hline Corydoras aff. paleatus & e,h,l & & & $x$ & \\
\hline Hoplosternum littorale (Hancock, 1828) & $a, b, c, d, e, f, g, h, i, j, k, l$, & $\mathrm{X}$ & & $\mathrm{X}$ & IS \\
\hline Hypophthalmus edentatus Spix \& Agassiz, 1829* & $\mathrm{a}, \mathrm{c}$ & & $\mathrm{X}$ & & RS \\
\hline Hypophthalmus marginatus Valenciennes, $1840^{*}$ & a & & $\mathrm{X}$ & & RS \\
\hline Hypophthalmus fimbriatus Kner, 1858 & a & & $\mathrm{X}$ & & RS \\
\hline Leiarius marmoratus (Gill, 1870)* & a & $\mathrm{X}$ & $x$ & & IS \\
\hline \multicolumn{6}{|l|}{ Cetopsidae } \\
\hline Cetopsis gobioides Kner, 1858 & $h, j, I$ & & $x$ & & $\mathrm{RS} / \mathrm{LI}$ \\
\hline \multicolumn{6}{|l|}{ Doradidae } \\
\hline Franciscodoras marmoratus (Lütken, 1874) & j & $x$ & & & IS \\
\hline \multicolumn{6}{|l|}{ Heptapteridae } \\
\hline Cetopsorhamdia iheringi Schubart \& Gomes, 1959 & $h, j$ & $\mathrm{x}$ & $\mathrm{X}$ & & LI \\
\hline Rhamdia quelen (Quoy \& Gaimard, 1824) & $a, g, h, I$ & $\mathrm{X}$ & & $\mathrm{X}$ & IS \\
\hline Rhamdia voulezi Haseman, $1911^{*}$ & $\mathrm{~h}$ & $\mathrm{X}$ & & & IS \\
\hline \multicolumn{6}{|l|}{ Loricariidae } \\
\hline Hypostomus isbrueckeri Reis, Weber \& Malabarba, 1990 & 1 & $\mathrm{X}$ & & & RS \\
\hline Loricariichthys anus (Valenciennes, 1835) & $f, g, h, I$ & $\mathrm{X}$ & $\mathrm{X}$ & & IS \\
\hline Loricariichthys platymetopon Isbrücker \& Nijssen, 1979 & g,h,l & $\mathrm{X}$ & $\mathrm{X}$ & & RS \\
\hline Rhinelepis aspera Spix \& Agassiz, 1829* & $h, j$ & $\mathrm{X}$ & & $\mathrm{X}$ & IS \\
\hline Pimelodidae & & & & & \\
\hline Brachyplatystoma filamentosum (Lichtenstein, 1819)* & $a, b, c$ & & $x$ & & \\
\hline Brachyplatystoma juruense (Boulenger, 1898)* & a & & $x$ & & \\
\hline Brachyplatystoma platynemum Boulenger, 1898 & a & & $x$ & & \\
\hline Brachyplatystoma rousseauxii (Castelnau, 1855) $^{*}$ & a & & $\mathrm{X}$ & & \\
\hline
\end{tabular}

HR: Hydrographic region of origin, $\mathrm{a}=$ Amazon, $\mathrm{b}=$ Western Northeast Atlantic, $\mathrm{c}=$ Eastern Northeast Atlantic, $\mathrm{d}=$ East Atlantic, $\mathrm{e}=$ Southeast Atlantic, $\mathrm{f}=$ South Atlantic, $\mathrm{g}=$ Paraguay, $\mathrm{h}=$ Paraná, $\mathrm{i}=$ Parnaíba, $\mathrm{j}=$ São Francisco, $\mathrm{k}=$ Tocantins-Araguaia and $\mathrm{i}=$ Uruguay; Larvae, $\mathrm{I}=$ Incomplete, without all larval stages and $\mathrm{C}=$ Complete, with all larval stages; $\mathrm{MU}=$ Methodology used to obtain the collection, $\mathrm{RS}$ = regressive series, $\mathrm{IS}=$ induced spawning and LI = live ichthyoplankton * commercial species. 
Table 1. Continued...

\begin{tabular}{|c|c|c|c|c|c|}
\hline \multirow{2}{*}{ Species } & \multirow{2}{*}{ HR } & \multirow{2}{*}{ Egg } & \multicolumn{2}{|c|}{ Larvae } & \multirow{2}{*}{ MU } \\
\hline & & & $\mathbf{L}$ & C & \\
\hline Brachyplatystoma tigrinum (Britski, 1981)* & a & & $\mathrm{X}$ & & \\
\hline Brachyplatystoma vaillantii (Valenciennes, 1840$)^{*}$ & $a, b, c$ & & $\mathrm{X}$ & & \\
\hline Hemisorubim platyrhynchos (Valenciennes, 1840$)^{*}$ & $\mathrm{a}, \mathrm{h}$ & $\mathrm{X}$ & & $\mathrm{X}$ & IS \\
\hline Iheringichthys labrosus (Lütken, 1874) & $g, h, I$ & & $\mathrm{X}$ & & RS \\
\hline Parapimelodus valenciennis (Lütken, 1874) & $\mathrm{g}, \mathrm{h}, \mathrm{I}$ & $\mathrm{X}$ & $x$ & & LI \\
\hline Pimelodus britskii Garavello \& Shibatta, 2007 & $\mathrm{~h}$ & & & $\mathrm{X}$ & RS \\
\hline Pimelodus maculatus Lacepède, 1803* & $g, h, j, l$ & $\mathrm{X}$ & $\mathrm{X}$ & & IS/RS \\
\hline Pimelodus ortmanni Haseman, 1911 & $\mathrm{~h}$ & $\mathrm{X}$ & & $\mathrm{X}$ & IS \\
\hline Platynematichthys notatus (Jardine, 1841)* & a & & & $\mathrm{X}$ & LI \\
\hline Pseudoplatystoma corruscans (Spix \& Agassiz, 1829)* & $g, h, j, I$ & $\mathrm{X}$ & & $\mathrm{X}$ & IS \\
\hline Pseudoplatystoma fasciatum (Linnaeus, 1766) ${ }^{*}$ & $\mathrm{a}, \mathrm{h}$ & $\mathrm{X}$ & & $\mathrm{X}$ & IS \\
\hline Pseudoplatystoma reticulatum Eigenmann \& Eigenmann, 1889* & $\mathrm{a}, \mathrm{h}$ & $\mathrm{X}$ & & $\mathrm{X}$ & IS \\
\hline Pseudoplatystoma tigrinum (Valenciennes, 1840)* & a & & $\mathrm{X}$ & & \\
\hline Sorubim lima (Bloch \& Schneider, 1801)* & $a, h, i$ & & & $\mathrm{X}$ & IS \\
\hline Sorubimichthys planiceps (Spix \& Agassiz, 1829)* & a & & $\mathrm{X}$ & & \\
\hline Steindachneridion melanodermatum Garavello, 2005* & $\mathrm{h}$ & $\mathrm{X}$ & & $\mathrm{X}$ & IS \\
\hline Steindachneridion parahybae (Steindachner, 1877)* & d,e & $\mathrm{X}$ & & $\mathrm{X}$ & IS \\
\hline Steindachneridion scriptum (Miranda Ribeiro, 1918)* & $h, \mathrm{l}$ & $\mathrm{X}$ & $\mathrm{X}$ & & IS \\
\hline Zungaro jahu (Ihering, 1898)* & g,h & & & $\mathrm{X}$ & IS \\
\hline \multicolumn{6}{|l|}{ Pseudopimelodidae } \\
\hline Lophiosilurus alexandri Steindachner, 1876* & j & $\mathrm{X}$ & & $\mathrm{X}$ & IS \\
\hline Microglanis eurystoma Malabarba \& Mahler, 1998* & I & & $\mathrm{X}$ & & RS \\
\hline Pseudopimelodus mangurus (Valenciennes, 1835) & $g, h, I$ & $X$ & $\mathrm{X}$ & & IS \\
\hline \multicolumn{6}{|l|}{ SYNBRANCHIFORMES } \\
\hline \multicolumn{6}{|l|}{ Synbranchidae } \\
\hline Synbranchus lampreia Favorito, Zanata \& Assumpção, 2005 & a & $\mathrm{X}$ & & $\mathrm{X}$ & \\
\hline \multicolumn{6}{|l|}{ TETRAODONTIFORMES } \\
\hline \multicolumn{6}{|l|}{ Tetraodontidae } \\
\hline Colomesus asellus (Müller \& Troschel, 1849) & $a, b, c$ & & $\mathrm{X}$ & & RS \\
\hline
\end{tabular}

HR: Hydrographic region of origin, $\mathrm{a}=$ Amazon, $\mathrm{b}=$ Western Northeast Atlantic, $\mathrm{c}=$ Eastern Northeast Atlantic, $\mathrm{d}=$ East Atlantic, $\mathrm{e}=$ Southeast Atlantic, $\mathrm{f}=$ South Atlantic, $\mathrm{g}=$ Paraguay, $\mathrm{h}=$ Paraná, $\mathrm{i}=$ Parnaíba, $\mathrm{j}=$ São Francisco, $\mathrm{k}=$ Tocantins-Araguaia and $\mathrm{i}=$ Uruguay; Larvae, $\mathrm{I}=$ Incomplete, without all larval stages and $\mathrm{C}=$ Complete, with all larval stages; $\mathrm{MU}=$ Methodology used to obtain the collection, $\mathrm{RS}=$ regressive series, IS = induced spawning and LI = live ichthyoplankton * commercial species.

\section{Discussion}

Studies with larvae of Brazilian freshwater fish are relatively recent. According to Bialetzki et al. (2016), the first studies were performed concomitantly in the Amazon and Paraná basins in the 1980s, resulting in descriptions of the early development of Semaprochilodus insignis (Jardine, 1841) (Araújo-Lima, 1985) and Catathyridium jenynsii (Günther, 1862), Hypophthalmus edentatus (Spix, 1829) and Plagioscion squamosissimus (Heckel, 1840) (Nakatani et al., 1997; 1998). However, a major breakthrough in research came in 2001 with the publication of several articles describing ichthyoplankton and especially the publication of the book Ovos e larvas de peixes de água doce: desenvolvimento e manual de identificação
(Nakatani et al., 2001), which included the morphological description of eggs, larvae and juveniles of 62 species present in several Brazilian watersheds. This book was responsible for the increase of in situ studies of the ichthyoplankton community, since the identification of the material was compromised by the lack of taxonomic support. In addition, it served as an incentive for contemporary researchers to produce new descriptions, as evidenced by the increase in the number of articles, but also the occurrence of dissertations/theses and chapters of books/books from this publication.

Nevertheless, the significant step taken in 2001, mainly by the team of PhD. Keshiyu Nakatani, has not yet resulted in a 'big step' for the effective 
development of the area, given the very low number of descriptions. There are 3,417 species of fish recorded in Brazilian continental waters (Froese \& Pauly, 2019) and only 140 or $4.1 \%$ had their early development known and described in the literature. Possibly, the incipient state of knowledge of ichthyoplankton in Brazil is mainly related to the difficulties inherent to its study, such as the large number of species with high similarity during early development, the small number of experts working in this area, the lack of resources and the limited number of teaching and research institutions working with ichthyoplankton.

The scarcity of studies that detail the morphological and meristic characteristics of eggs and larvae, as well as identification keys negatively influence the surveys, monitoring and management studies. Many studies carried out with an ichthyoplankton community in different environments face the difficulty of identification at lower taxonomic levels and an expressive portion of this community is categorized only at the family level, mainly Anostomidae, Characidae and Pimelodidae (Oliveira \& Ferreira, 2008; Tondato et al., 2010; Ziober et al., 2012; Lima et al., 2013; Ziober et al., 2015; Silva et al., 2017). This is especially severe in megadiverse basins such as the Amazon and São Francisco, where the scarcity of this type of study is remarkable if we observe the ratio between the number of species studied/the number of species present in the basin.

It is clear the need for studies that characterize embryonic and larval development, detailing morphological characteristics such as body shape, pigmentation, measurements and counts that help identify species, as well as comparative studies showing differences between similar species. This approach cannot be restricted to species of commercial interest which, because of their greater ease in obtaining the breeders has presented a greater number of publications. Also, because the absence of a study of fish of lesser commercial importance influences the accuracy in the identification of all species due to the "doubt" generated by not knowing how the eggs and larvae of these species are and whether they can be the same or similar to those already known in the literature.

Among the tools that can be used to conduct egg and larval characterization studies, we highlight three: 1. The use of induced reproduction; 2. The methodology of live ichthyoplankton
(Reynalte-Tataje \& Zaniboni-Filho, 2008) and 3. The regressive series method (Ahlstrom \& Moser, 1976). The first consists of obtaining a breeding stock and the use of gonadotropic hormones or environmental stimuli that induce reproduction. This tool, despite being used constantly in species of commercial interest, can be used in other species, perhaps with some variants in the hormonal dosages and stimuli.

The second method consists of the capture of the eggs and/or larvae in the environment, followed by the maintenance of the individuals in the laboratory during the development. This allows the characterization of all the stages until the juvenile period. In this case, it is necessary the availability of infrastructure to receive the organisms, the advanced knowledge of the researchers in incubation and fish larviculture techniques and the use of anesthetics, such as clove oil, to be able to carry out the characterization of the species in each stage of the embryonic and larval development, without the need to sacrifice the animal. This method can be an efficient alternative for rare species where there is the difficulty of making the breeding stock or even at institutions that lack facilities for their maintenance. A great problem for the execution of methods 1 and 2 is the feeding of larvae, since little is known about this aspect, mainly for the first stages of development. A factor that worsens when some species during larval metamorphosis also change the feeding behavior (Reynalte-Tataje \& Zaniboni-Filho, 2008), or due to the difficulty of obtaining the food, such as the larviculture of Salminus brasiliensis (Cuvier, 1816), which only eats larvae of fish with great voracity, and can eat for example on the fifth day of life more than 41 larvae (Weingartner et al., 2003).

The regressive series method is also widely used since it takes advantage of the organisms collected in nature in the survey/monitoring studies to assemble a collection. This method is particularly useful for species with well-defined morphological and meristic characteristics, but it is not useful when the species are very similar or when the studied environment presents a very great diversity of species, as in the Amazon. Moreover, this method depends on a large number of samples and only applies to larvae and not to eggs, which makes unfeasible a complete collection of eggs and larvae. The combined use of these three methods can be a valuable tool for making a complete sequence of species development, especially highlighting the 
combined use of methods 2 and 3, since the use of method 1 generally tends to give a complete sequence of all without the need of others.

In view of the difficulties presented, a complementary alternative for the identification of eggs and larvae is the use of genetic methods (Becker et al., 2015; Frantine-Silva et al., 2015; Almeida et al., 2018). In the absence of studies that characterize eggs and larvae in a particular environment or in the presence of rare and cryptic species that are morphologically similar but genetically distinct or for the identification of larval yolk, at which many species share meristic and morphometric characteristics, where the use of the traditional method of identification is limited, the use of genetic methods such as DNA barcoding cannot be ruled out. This technique, based on the DNA barcode that evaluates the genetic distances between sibling species (Pradhan et al., 2015), can be an important tool when the task of identifying ichthyoplankton organisms is not feasible or there is a very low degree of confidence in the traditional method.

Finally, we can conclude that although the ichthyoplankton area is extremely important in fish ecology, its keystone, which is the characterization of eggs and larvae, is still little studied, being limited mainly to the basins of the Plata: Paraná, Paraguay and Uruguay and fish of commercial importance. Thus, actions to solve the question of knowledge about the early ontogeny of the continental species of Brazil are urgent and relevant for the development of satisfactory research on fish ecology and fisheries biology. As an immediate action we suggest (1) a joint effort among the researchers of the area to increase the number of publications on the early ontogeny, starting with those that are known, but are not described in the literature; and in the medium term: (2) Creation of programs and/or partnerships between research institutions and agencies or companies working with induced reproduction and larviculture, seeking to establish protocols to obtain collections, mainly of species without commercial interest; (3) Inclusion of genetic analyses in projects developed in the field; and (4) Training of new researchers, either through undergraduate or graduate academic work or mini-courses or courses. With the fulfillment of these actions, our expectation is an expressive increase in the number of publications in the coming years, as well as in the number of researchers who want to explore this area still poorly explored in Brazil.

\section{References}

AHLSTROM, E.H. and MOSER, H.G. Eggs and Larvae of Fishes and their role in systematic investigations and in fisheries. Revue des Travaux de l'Institut des Pêches Maritimes, 1976, 40(1), 379-398.

ALMEIDA, F.S., FRANTINE-SILVA, W., LIMA, S.C., GARCIA, D.A.Z. and ORSI, M.L. DNA barcoding as a useful tool for identifying non-native species of freshwater ichthyoplankton in the neotropics. Hydrobiologia, 2018, 817(1), 111-119. http://dx.doi. org/10.1007/s10750-017-3443-5.

ARAÚJO-LIMA, C. Aspectos biológicos de peixes amazônicos. V. Desenvolvimento larval do jaraqui-escama grossa, Semaprochilodus insignis (Characiformes, Pisces) da Amazônia Central. Revista Brasileira de Biologia, 1985, 45(4), 423-431.

BALDISSEROTTO, B. and GOMES, L.C. Espécies nativas para piscicultura no Brasil. Santa Maria: Editora da UFSM, 2010.

BECKER, R.A., SALES, N.G., SANTOS, G.M., SANTOS, G.B. and CARVALHO, D.C. DNA barcoding and morphological identification of neotropical ichthyoplankton from the Upper Paraná and São Francisco. Journal of Fish Biology, 2015, 87(1), 159-168. http://dx.doi.org/10.1111/ jfb.12707. PMid:25988313.

BIALETZKI, A., ORSI, M.L. and GARCIA, D.A.Z. O estudo de ovos e larvas de peixes. In: M.L. ORSI, A.C. SWARÇA, A. CLARO-GARCÍA, N.C. VIANNA, D.A.Z. GARCIA and A. BIALETZKI, eds. Ovos, Larvas e Juvenis dos peixes da bacia do Rio Paranapanema: uma avaliação para a conservação. 1st ed. São Paulo: Triunfal Gráfica e Editora, 2016, pp. 17-24.

BRASIL. Ministério do Meio Ambiente. Conselho Nacional de Recursos Hídricos. Resolução no 32, de 15 de outubro de 2003. Considerando a necessidade de se implementar base de dados referenciada por bacia, em âmbito nacional, visando a integração das informações em recursos hídricos. Diário Oficial da União [da] República Federativa do Brasil. Brasília, DF, 17 dez. 2003.

FRANTINE-SILVA, W., SOFIA, S.H., ORSI, M.L. and ALMEIDA, F.S. DNA barcoding of freshwater ichthyoplankton in the Neotropics as a tool for ecological monitoring. Molecular Ecology Resources, 2015, 15(5), 1226-1237. http://dx.doi. org/10.1111/1755-0998.12385. PMid:25655460.

FRICKE, R., ESCHMEYER, W.N. and VAN DER LAAN, R. Catalog of fishes: Genera, Species, References. San Francisco: California Academy of Sciences, 2019 [viewed 22 Mar. 2019]. Available from: http:// researcharchive.calacademy.org/icthyology/catalog/ fishcatmain.asp.

FROESE, R. and PAULY, D. Fishbase. World Wide Web electronic publication Version (02/2019) [online]. 
Stockholm, Sweden: Fishbase, 2018 [viewed 10 Nov 2018]. Available from: http://www.fishbase.org.

GODINHO, H.P. Estratégias reprodutivas de peixes aplicadas à aqüicultura: bases para o desenvolvimento de tecnologias de produção. Revista Brasileira de Reprodução Animal, 2007, 31(3), 351-360.

LIMA, A.F., MAKRAKIS, M.C., SILVA, P.S., AZEVEDO, A.V., MAKRAKIS, S., ASSUMPÇÃO, L., ANDRADE, F.F. and DIAS, J.H.P. Padróes de distribuição e ocorrência espaço-temporal de ovos e larvas de peixes nos rios Pardo e Anhanduí, bacia do alto rio Paraná, Brasil. Revista Brasileira de Biociências, 2013, 11(1), 7-13.

NAKATANI, K., AGOSTINHO, A.A., BAUMGARTNER, G., BIALETZKI, A., SANCHES, P.V., MAKRAKIS, M.C. and PAVANELLI, C.S. Ovos e larvas de peixes de Água doce: desenvolvimento e manual de identificação. Maringá: Eduem, 2001.

NAKATANI, K., BAUMGARTNER, G. and BAUMGARTNER, M.S. Larval development of Plagioscion squamisissimus (Heckel) (Perciformes, Sciaenidae) of Itaipu Reservoir (Paraná River, Brazil). Revista Brasileira de Zoologia, 1997, 14(1), 35-44. http://dx.doi.org/10.1590/S010181751997000100003

NAKATANI, K., BAUMGARTNER, G. and LATINI, J.D. Morphological description of larvae of the Mapará Hypophthalmus edentatus (Spix) (Osteichthyes, Hypophthalmidae) in the Itaipu reservoir (Parana River, Brazil). Revista Brasileira de Zoologia, 1998, 15(3), 687-696. http://dx.doi. org/10.1590/S0101-81751998000300013.

OLIVEIRA, E.C. and FERREIRA, E.J.G. Spawning areas, dispersion and microhabitats of fish larvae in the Anavilhanas Ecological Station, rio Negro, Amazonas State, Brazil. Neotropical Ichthyology, 2008, 6(4), 559-566. http://dx.doi.org/10.1590/S167962252008000400003

PRADHAN, V., KAMBLE, Y., LADNIYA, V. and MOGUL, M.A. overview of species identification by DNA barcoding. International Journal of Current Microbiology and Applied Sciences, 2015, 4(4), $127-$ 140.

REIS, R.E., KULLANDER, S.O. and FERRARIS, C.J. Check list of the freshwater fishes of South and Central America. Porto Alegre: Edipucrs, 2003.

REYNALTE-TATAJE, D.A. and ZANIBONI-FILHO, E. Biologia e identificação de ovos e larvas de peixes do alto rio Uruguai, Brasil. In: ZANIBONI-FILHO,
E. and NUNERR, A.P.O., eds. Reservatório de Itá: Estudos ambientais, desenvolvimento de tecnologias de cultivo e conservação da Ictiofauna. 1st ed. Florianópolis: Editora da UFSC, 2008, pp. 139-154.

SANTIN, M., BIALETZKI, A. and NAKATANI, K. Mudanças ontogênicas no trato digestório e dieta de Apareiodon affinis (Steindachner, 1879) (Osteichthyes, Parodontidae). Acta Scientiarum. Biological Sciences, 2009, 26(3), 291-298.

SILVA, J.C., ROSA, R.R., GALDIOLI, E.M., SOARES, C.M., DOMINGUES, W.M., VERÍSSIMO, S. and BIALETZKI, A. Importance of dam-free stretches for fish reproduction: The last remnant in the Upper Paraná River. Acta Limnologica Brasiliensia, 2017, 29(0), e106. http://dx.doi.org/10.1590/s2179$975 \times 10216$.

TONDATO, K.K., MATEUS, L.A.F. and ZIOBER, S.R. Spatial and temporal distribution of fish larvae in marginal lagoons of Pantanal, Mato Grosso State, Brazil. Neotropical Ichthyology, 2010, 8(1), 123-134. http://dx.doi.org/10.1590/S167962252010005000002 .

VALLADÃO, G.M.R., GALLANI, S.U. and PILARSKI, F. South American fish for continental aquaculture. Reviews in Aquaculture, 2018, 10(2), 351-369. http:// dx.doi.org/10.1111/raq.12164.

WEINGARTNER, M., REYNALTE-TATAJE, D.A and ZANIBONI-FILHO, E. Determinacion del consumo diário de larvas forrajeras de curimbatá (Prochilodus lineatus) por larva de dourado (Salminus brasiliensis) durante la fase inicial de larvicultura. In: VII Simpósio Colombiano de Ictiologia. Monteria, Colombia: Universidad de Córdoba, 2003, pp. 96-96.

ZIOBER, S.R., BIALETZKI, A. and MATEUS, L.A.F. Effect of abiotic variables on fish eggs and larvae distribution in headwaters of Cuiabá River, Mato Grosso State, Brazil. Neotropical Ichthyology, 2012, 10(1), 123-132. http://dx.doi.org/10.1590/S167962252012000100012 .

ZIOBER, S.R., REYNALTE-TATAJE, D.A. and ZANIBONI-FILHO, E. The importance of a conservation unit in a subtropical basin for fish spawning and growth. Environmental Biology of Fishes, 2015, 98(2), 725-737. http://dx.doi. org/10.1007/s10641-014-0307-y.

Received: 28 April 2019

Accepted: 12 March 2020 